\title{
Aportes de Polo para superar las perspectivas reductivas del homo oeconomicus de la escuela científica de F. Taylor
}

\author{
Polo's Contributions to overcome the \\ Reductive Perspectives of Homo \\ Oeconomicus from the Scientific School \\ ofF. Taylor
}

\section{SILVIA CAROLINA MARTINO \\ silviamartin0500@gmail.com \\ Universidad Austral (Argentina). \\ ORCID: 0000-0003-2073-8064}

\section{Resumen}

Este artículo intenta exponer algunas claves de Polo sobre la empresa, como lugar de cambio social y de esperanza para el crecimiento de la persona humana. Entendemos que los aportes antropológicos de su planteamiento pueden ser supuestos para superar la crisis actual de lo que hoy se entiende por Acción Directiva o Management. Para exponer estas claves se plantea hacer un recorrido a través de las distintas escuelas de Management en diálogo con sus autores y propuestas para intentar superar la perspectiva reductiva que tienen. Se busca exponer el aporte de Polo que puede añadirse o lo que sugiere rectificar para superar las miradas reductivas de la empresa y del hombre trabajando en ella. En esta comunicación se expondrá la denuncia poliana frente al planteamiento del homo oeconomicus que plantea la primera escuela de management, llamada científica de F. Taylor.

Palabras clave: Empresa y Antropología, Crisis del pensamiento sobre Management, Homo Oeconomicus en F. Taylor.

\section{Summary}

This communication tries to expose some keys of Polo about the company, as a place of social change and of hope for the growth of the human person. It is understood that the anthropological contributions of his approach can be assumed to overcome the current crisis of what is today understood by Directive Action or Management. To expose these keys, it is proposed to take a tour through the different Management schools in dialogue with their authors and proposals to try to overcome the reductive perspective they have. It seeks to expose Polo's contribution that can be added or what he suggests to rectify to overcome the reductive views of the company and the man working in it. In this communication, the Poliana complaint against the homo oeconomicus approach that the first so-called scientific management school of F. Taylor raises will be exposed.

Keywords: Business and Anthropology, Crisis of thought about Management, Homo oeconomicus in F. Taylor. 


\section{INTRODUCCIÓN}

La importancia de repensar la actividad empresarial y la empresa como tal es importante, pues "El management moderno ha dado mucho, pero también se ha llevado mucho, y continúa haciéndolo. Tal vez haya llegado el momento de renegociar la oferta. Debemos aprender a coordinar los esfuerzos de miles de individuos sin crear una jerarquía opresiva de supervisores; a controlar los costes con firmeza sin asfixiar la imaginación humana, y a crear organizaciones donde la disciplina y la libertad no se excluyen mutuamente. En este nuevo siglo debemos esforzarnos por trascender las relaciones de intercambio aparentemente inevitables que forman el incómodo legado del management moderno" .

Como indica Corcuera ${ }^{2}$ y también Martínez Echevarría ${ }^{3}$, se observa que la visión moderna de las organizaciones ha desarrollado un cuerpo de bibliografía sobre el análisis empírico de los directivos "exitosos" como único fundamento para entender el trabajo directivo, y su repercusión inmediata en los programas de formación en dirección de empresas. Este parecer coincide con lo expuesto por Martínez Echevarría: "Si se presta atención a lo que se ha publicado y enseñado en los últimos años sobre qué es una empresa y cuál es el sentido de la actividad que desempeñan sus directivos, una de las cosas que más sobresale es la existencia de un fuerte dualismo, casi se podría decir de una esquizofrenia. Mientras que desde el punto de vista teórico -el propio de los economistas- la empresa se entiende como una función abstracta de producción que siempre permite una solución óptima, desde un enfoque práctico -el propio de los directivos- la empresa se presenta como un conjunto de problemas concretos y cambiantes, que hay que ir resolviendo en el día a día, sin arreglo a una teoría de la empresa"4. Además, se suelen trabajar los temas vinculados a lo empresarial de un modo hiperespecializado y esto va en detrimento de una mirada integral de la organización empresarial. Lo que se puede comprobar al investigar los contenidos de los Programas de Posgrados es que la ciencia hoy avanza en silos. De hecho, hay muy pocos autores contemporáneos que traten sobre el management y la gerencia. Esta ausencia de pensadores de relevancia que en sus planteamientos aborden la organización empresarial y la acción directiva de un modo integrado lo expondremos en el punto referido a los autores y escuelas. Tanto Gary Hamel ${ }^{5}$ como Henry Mintzberg,

1 Hamel, G. y Breen, B. (2008), p. 25.

2 Cfr. Corcuera, J.P. (2001), p. 11, p. 42 y p. 51.

3 Martínez Echevarría, M. A. (2015), p. 8.

4 Martínez-Echevarría, M. A. (2015), p. 5. 
desde otra perspectiva ${ }^{6}$, plantean la misma cuestión referente a la falta de autores que sostengan posiciones que difieren esencialmente de lo que plantearon los clásicos.

La bibliografía sobre temas de empresa actualmente avanza en nichos ${ }^{7}$, con una fuerte dosis matemática, de casuística y modas. Muchos de estos factores que mencionamos han conducido a que se tenga una mirada atomizada, parcial y sesgada de la empresa, de la tarea del directivo y, en definitiva, de la persona en las organizaciones. Nos parece que, en última instancia, lo que no acaba de estar claro es lo que se entiende por directivo y por empresa. Esto responde a una mirada reductiva, porque se piensa en el mejoramiento de productos, innovación en logística, innovación en servicios, sin reparar que realmente lo que hace falta es que haya innovación en la gestión empresarial, porque conlleva un beneficio a mediano y largo plazo. Es más importante pensar en cómo se hacen las cosas, cómo se gestiona la compañía que lo que se vende. Por eso, frente a los retos de las grandes cuestiones -barreras de entradas a la industria que ya no existen, dificultades muy grandes para proteger la propiedad intelectual, ciclos de vida de los productos muy cortos, consumidores que controlan y poseen toda la información, categorías enteras o rubros enteros en crisis-, las herramientas académicas o teóricas para manejar estos problemas son viejas, algunas tienen casi una centuria ${ }^{8}$.

$\mathrm{Al}$ estudiar a los autores más destacados sobre el trabajo directivo ${ }^{9}$ podremos advertir que se suele entender que el progreso sigue en gran parte el influjo de un concepto reductivo de racionalidad, pragmática, suscrita por el cientificismo positivista ${ }^{10}$. Nuestro empeño pasa por estudiar la conexión que

5 Hasta el 2008 Hamel sostiene que han pasado 40 años en los que no ha habido pensadores que planteen innovaciones para la Gestión. Cfr. Hamel, G.y Brren, B. (2008), pp. 22-30, y pp. 36-37.

6 Mintzberg, H.; Ahlstrand, B. y Lampel, J. (1999).

7 Cfr. Corcuera, J. P. (2001), p. 113.

8 Cfr. Hamel, G. y Brren, B. (2008), pp. 25-26.

9 La selección de los autores o escuelas ha sido el resultado de un trabajo previo de búsqueda en los Manuales más utilizados en las Carreras de Empresariales. Hay una coincidencia que llama la atención entre los diferentes textos.

10 Sellés expone que "el método analítico (propio de las ciencias positivas) no es procedente para desentrañar lo humano, ni lo que pertenece a la esencia ni lo que pertenece al acto de ser, a la persona". No es posible trocear por partes y analizar para luego reunirlas nuevamente pues su sentido es como suele decirse que el todo es mayor de la suma de las partes. Así se comprende que lo que refiere a las manifestaciones humanas de la esencia del hombre "es mejor que se estudien con un método reunitivo, ver la hilazón que guardan las diversas manifestaciones humanas entre sí (su jerarquía o dualización o vinculación)". Por eso conviene proceder "sistémicamente, esto es, aunando las diversas facetas de lo humano a fin de explicar el engarce de todas las manifestaciones humanas" así "se saca partido del método sistémico para dilucidar la esencia humana”. Sellés, J.F (2011), p. 511. 
existe entre teoría y práctica ${ }^{11}$, o sea, ciencia pura y ciencia aplicada. Intentaremos abordarlo a través del trabajo directivo, entendiendo que ambas están relacionadas y no se pueden separar. En su original concepción Aristóteles plantea que la ciencia ha de tener base empírica y estructura formal o deductiva. Ambas habrán de estar bien fundamentadas, ser rigurosas. Para que la conexión pueda hacerse científicamente, conviene utilizar una disciplina que pueda dar cuenta de los fenómenos empíricos como manifestaciones de las acciones humanas. Sobre esto Polo escribe que "los hombres que se centran en la práctica se sorprenden al ver que la gente se mueve por ideas y que esas ideas afectan a sus negocios. ¿Qué tienen que hacer las ideas en el mundo de los negocios? Es claro que tienen que ver con él y que lo favorecen o perturban. Es un rasgo de la sociedad occidental" 12 .

Se trata de comprender que el directivo "es una persona que sabe descubrir y aprovechar alternativas y, por consiguiente, cambiar. La empresa (se entiende como) un proceso dinámico"13, un proceso que es abarcativo de la persona en todas sus manifestaciones. Por eso, nos parece que lo conveniente es que los planteamientos sobre el directivo y sobre el trabajo empresarial sean integrales, reunitivos, esto es, que consideren primero el aspecto antropológico, personal; luego el familiar ${ }^{14}$ como raíz de la ética ${ }^{15}$; tercero, el aspecto ético vinculado al desarrollo de las virtudes en su voluntad; cuarto, el empeño para adquirir los hábitos intelectuales; finalmente, todos los temas que se vinculan al ordenamiento de los medios, el ambiente de la empresa, los departamentos funcionales, etc. Esto implica dar prioridad a lo más radical para luego poder anclar la organización en el verdadero dinamismo innovador propio de las personas.

A este respecto encontramos muy apropiado el planteamiento y las preguntas que presenta Gary Hamel cuando cuestiona la visión clásica de la empresa, la cual podría presentarse con las diversas respuestas a esta pregunta: ¿Cómo lograr que los seres humanos hagan lo mismo todos los días de la misma manera y cada vez con mayor productividad? O, dicho de otro modo: ¿cómo convertir seres humanos libres y pensantes en algún tipo de robots pro-

11 Polo, L. (1996), p. 117.

12 Polo, L. (2015), p. 51.

13 Polo, L. (1997), p. 102.

14 Polo, L. (2015), p. 459.

15 En tanto que implica el respeto por la persona en su vocación o sentido familiar. En relación al aspecto ético, Cuando la empresa no encuadra bien lo familiar, tampoco puede encuadrar lo Antropológico personal y lo ético humano. Sellés, J.F. (2013), p. 139. Cfr. Chinchilla, N. (2006). 
gramable más o menos sofisticados? Hamel indica que el haber planteado las organizaciones en términos de eficacia y productividad ha conducido a que las organizaciones cada vez sean menos humanas que los humanos que trabajan en ellas. En algún sentido este autor sostiene que ha habido logros ciertos en estos años ${ }^{16}$. Sin embargo, la eficiencia, la disciplina, el cumplimiento, la concentración, sin más, no darán ninguna ventaja competitiva, ningún diferencial ni a la organización ni a las personas que en ella se desempeñan ${ }^{17}$. Hamel defiende que al hacer del trabajo un commodity, estamos haciendo un commodity de la gente y olvidamos que son seres humanos con un gran potencial creativo. Por esto, la pregunta que plantea es: ¿cómo aumentar el contenido creativo de cada trabajo liberando capacidad creativa de cada individuo? Si estamos en un punto de inflexión histórico ${ }^{18}$ en el que hay que replantearse la organización, hay que cambiar el modo de entender la innovación, el cambio $\mathrm{y}$, en definitiva, la gestión del management; lo que parece oportuno es que habrá que innovar agregando valor real. En concreto, el punto que nos parece más interesante de toda su propuesta es el último de los cinco asuntos fundamentales que indica: la búsqueda de una fundamentación antropológica ${ }^{19}$. Justamente en éste, pues el autor reconoce que de lo contrario su propuesta es vulnerable, es donde consideramos que el planteamiento antropológico de Leonardo Polo podría ayudarnos a intentar hacer algún aporte o aproximación a una fundamentación antropológica personal que permita encontrar el equilibrio entre el control y la libertad, para la correcta gestión de cualquier estrategia empresarial, y otorgue también claves para un anclaje ético humano que permita ese renacimiento moral desde dentro de los miembros de la organización. Esto permite comprender que la innovación se funda en la persona misma que se da en la actividad humana libre.

Esta comunicación intenta exponer algunas claves de Polo sobre la empresa, como lugar de esperanza para el crecimiento de la persona humana, porque sus aportes antropológicos son cuestión central para superar la crisis actual de nuestra sociedad. El intento se llevará a cabo intentando hacer un recorrido a través de las distintas escuelas de Management y cómo el aporte de Polo puede añadir o rectificar algunos aspectos para superar las miradas reductivas de la empresa y del hombre en ella. En esta comunicación se expon-

16 Cfr. Hamel, G. y Breen, B. (2008), p. 14, p. 23 y p. 24.

17 Cfr. Hamel, G. y Breen, B. (2008), p. 24.

18 Hamel, G. comenta en sus últimos dos libros, (2008) y (2012), que "todo ha entrado en bajada".

19 Cfr. Hamel, G. (2012). 
drá el diálogo entre Polo y F. Taylor. El estudio queda abierto a proseguirse buscando poner en diálogo a Polo con los autores que se ha concluido como más representativos ${ }^{20}$. La propuesta de poner en diálogo a una serie de autores a la selección de esos autores y a la posibilidad de tratar sobre los temas de relevancia para la Disciplina del Management, el trabajo, la libertad, el perfeccionamiento de la persona en el trabajo, etc. sobre los Polo plantea supuestos que pueden superar esta crisis y brindar fundamento.

Si no logramos alcanzar el verdadero saber empresarial, el del sentido personal de la actividad libre de cada quién ante la totalidad de lo real, se carece de un conocimiento global, sin fragmentaciones, con una visión integral que lo motive a involucrarse en su aprendizaje y crecimiento para 'ser más, haciendo'. Si la formación que se recibe es restringida, reductiva, dificulta que cada quien, desde el lugar que ocupa, sea un ser íntegro, atento a las problemáticas sociales y capacitado para actuar con resolución. Un ciudadano que logre ser un transformador positivo de la sociedad. Los futuros empresarios serán los actores claves en la empresa como 'directivos', buscarán que se desarrolle la empresa en forma genuina a medida que se humanizan y personalizan quienes en ellas trabajan. Esto sustenta la tesis de plantear a la empresa como una de las tres claves de cambio y esperanza de la sociedad actual.

\section{ALGUNOS APORTES CLAVES DE POLO}

\section{El hombre como perfeccionador perfectible.}

En el ámbito de la empresa, gran parte de los Directivos poseen un radio de intereses muy limitado, acotado a meros propósitos de maximización de los beneficios. Esto es una consecuencia lógica y esperable, pues los manuales de Management están inspirados en los 'grandes padres de las teorías' que han despojado a la actividad empresarial de sus valores humanos profundos ${ }^{21}$, sus abordajes han sido insuficientes. La empresa es una organización humana, dirigida por personas que deciden sobre acciones de personas y buscan satisfa-

20 Se ha realizado una selección vinculada con los autores que los Manuales de Management coinciden en considerar como los más representativos. Sobre el análisis del status quo de la disciplina. Cfr. Martino, Silvia C. (2018), pp. 308 y ss. Además, los Manuales de Management en uso coinciden en quiénes son los autores más representativos.

21 Cfr. Martínez Echevarría, M.A. (2001) y (2003).

22 Polo, L. (2016), p. 102. 
cer necesidades múltiples de las personas. Sin embargo, si las organizaciones pierden su sentido humano profundo de cohesión, de vinculación, de innovación, de desarrollo social y las acciones propias del hombre no se entienden como las de un "perfeccionador perfectible"22; con esta pérdida, toda la sociedad pierde. En algún lugar de este camino se ha perdido al hombre y a la persona humana, la visión antropológica que explica el sentido.

\section{La ética y el trabajo}

¿Cómo encontrar el sentido profundo de lo manifestativo humano? Queremos aprovechar los hallazgos de Polo en su planteamiento antropológico para rectificar o añadir a estas miradas sobre la empresa y sus elementos. En definitiva, algunos supuestos que puedan ayudarnos a superar la miopía, esta especie de "pérdida de altura y con ella el hombre que no tiene norte y por tanto no puede conseguir un futuro mejor." ${ }^{23}$ Esto es que la crisis de dirección de nuestras sociedades está en quienes las dirigen. No se considera que sobre todo acto humano uno responda por ellos, y que son las acciones humanas las que perfeccionan a la persona o a las organizaciones o la envilecen. Esto significa que hay "una idea equivocada de la ética" ${ }^{24}$ que nos circunda y que tienen muchos directivos. "La ética (...) es el tema del perfeccionamiento humano. Correlativamente se puede describir la ética como el modo de incrementar la tendencialidad del ser humano. (...) El hombre es ético cuando sus tendencias son cada vez más fuertes. Esta es una prueba de que el hombre desarrolla su vida éticamente. ¿Qué quiere decir que el hombre se perfecciona irrestrictamente? Que cada vez tiende a más, que cada vez es más fuerte" ${ }^{25}$.

Nos suele resultar complejo enlazar los aspectos de nuestro hacer con la integridad de lo manifestativo humano, más en concreto, con la ética -disciplina capaz de vincular ordenadamente las diversas manifestaciones humanas-. Manejamos técnicas, modelos, etc. pero desconocemos sobre quienes hacen distintas nuestras organizaciones, las personas. La antropología tiene un valor directo para la vida, de cómo se entienda el hombre dependen las actitudes y el desarrollo de su actividad, en nuestro caso su actividad de Gestión o acción empresaria Directiva. Cuando se entiende al hombre así, es posible plantear de manera positiva su modo de relacionarse con el entorno a través del trabajo. Y

23 Polo, L. (2016), p. 87.

24 Polo, L. (2016), p. 102.

25 Polo, L. (2016), p. 103. 
así el trabajo es fundamental porque es el enlace entre medios (propios de la economía y la gestión) y fines, saber y virtud en un plexo de medios.

\section{Las relaciones de personas mediadas por el dinero.}

Si la gestión de empresas trata de relaciones entre personas, con un intercambio de cosas mediado por el dinero, será relevante comprender quién y cómo es ese además -persona- que coexiste y aporta en forma inagotable y qué significa el dinero. La persona es coexistencia aportante, que da, que acepta, que intercambia, pero que no se agota en ese intercambio. El dinero no es fin, sino medio. Como tal se puede emplear para diversos fines. Todo lo que es medio respecto de un fin es inferior al fin. A su vez, los fines son diversos entre sí. Como en la realidad no todo tiene el mismo valor, la distinción entre los fines será también jerárquica. $\mathrm{O}$ sea, no es lo mismo usar el dinero para satisfacer los deseos placenteros individuales que para generar más trabajo para la empresa o de cara a favorecer el bien común. La pregunta que corresponde hacernos ahora es: ¿cómo debe usar el dinero el directivo? Para Polo, "el dinero, en esencia, para un señor que manda es esto: aquello que cuando se tiene posibilita el trabajo, es decir, el dinero que se tiene o que se recibe es trabajo en potencia. Cuando el dinero se emplea en el trabajo tiene una rentabilidad, es decir, el dinero se repone en ese mismo momento"26. En épocas de crisis, puede ser orientarse en primer a acordar entre todos los miembros de la empresa la reducción de los salarios y no apelar al recurso a los despidos.

"El primer cometido del capital es convocar al trabajo, es hacer trabajar, pero bien entendido, de tal manera que ese dinero siempre se transforme en trabajo potencial, es decir, que asegure la supervivencia del trabajo. Ése es el sentido estricto de la palabra inversión. La inversión es aquella función del dinero según la cual del trabajo en potencia se pasa al trabajo en acto, y del trabajo en acto se pasa otra vez al trabajo en potencia. Por eso, se puede decir que uno que manda es aquel que respecto del dinero no tiene la misma mentalidad de los procesos tecnológicos" ${ }^{27}$. El primer cometido del dinero es el referido al trabajo de los trabajadores y superior a éste es el bien común suyo, el de sus familias y el de la sociedad, y este bien no se reduce a dinero, a productos materiales y a trabajo, sino que es, sobre todo, de índole humanizante: la adquisición de hábitos intelectuales y virtudes en la voluntad. En este sen-

26 Polo, L. (2015), p. 418.

27 Polo, L. (2015), p. 418. 
tido, el dinero tiene sentido ético ${ }^{28}$. En caso contrario, tendría exclusivamente un sentido pragmático.

\section{La persona como fecunda y difusiva.}

Otro de los puntos en los que Polo otorga claves se vincula con el entendimiento de la persona como fecunda y difusiva. La coexistencia entre personas, el dar y aceptar, el dar aceptado es mucho más fecundo cuando nos referimos a personas que al referirnos a la productividad de una acción que tiene sus efectos en la naturaleza. Donde verdaderamente se da la difusión de ser es en la coexistencia entre personas. Si de ordinario miramos la dicotomía como distribución de bienes escasos, conviene mirar al productor, que siempre busca más, dar más, innovar más, porque lo efusivo que produce y lo que continúa el bien es el ser personal. Estas ideas sobre los trascendentales personales, sobre la fecundidad del ser personal hacen que podamos considerar el calado de la empresa como manifestación humana. En la empresa quien logra productividad es la persona, esto concluye con la difusión del bien en su comunidad, y allí los se logra que todos ganen. A todo esto se concluye cuando la persona es vista como ser donal (que aporta) y ser coexistente (que intercambia con los demás).

Con estos aportes de Polo es posible remarcar que insistir en la crisis en la que estamos inmersos, es importante, pero no puede asfixiar la posibilidad de enfrentar la cuestión y encontrar salidas superadoras con estas claves que hemos mencionado.

\section{UN DIÁLOGO CONVENIENTE ENTRE LOS AUTORES DE MANAGEMENT Y POLO.}

\section{La denuncia poliana ante el homo oeconomicus de la escuela científica(F.Taylor)}

Las concepciones más reconocidas sobre las empresas y la forma de concebir su dirección, surgen del estudio (análisis) de los problemas reales que

28 "El que ve al dinero como un modo de convocar, de hacer posible que la gente trabaje, se da cuenta del valor ético que tiene el trabajo. El dinero tiene valor ético si empleándolo se consigue que el trabajo en potencia se mantenga y aumente. Es decir que la capacidad de convocatoria para trabajar sea cada vez mayor". Polo, L. (2015), p. 418. 
fueron apareciendo en las grandes organizaciones, sobre todo con los propios trabajadores (mayormente obreros). Probablemente los autores más representativos e influyentes de la llamada concepción clásica del management sean Frederick Taylor y Henri Fayol. Sin embargo, dichos autores presentaron un conjunto coherente de ideas y principios que habían germinado y se habían cultivado en Gran Bretaña y Estados Unidos a lo largo del s. XIX. Se comienza a hablar de esta disciplina como "enfoques" o "escuelas de management" desde los escritos de F. W. Taylor (20 de marzo de 1856-21 de marzo de 1915) ${ }^{29}$ en Estados Unidos y H. Fayol (1841-1925) en Francia.

Polo, en relación con los primeros avances de organización empresarial, explica que hay "un aspecto decisivo de la penuria organizadora y es la ausencia de organización del trabajador industrial. Por lo pronto, la industria se desentiende de la organización, y el obrero no se integra en ella de un modo formal sino material: emplea su esfuerzo en una estructura formal a priori; es un productor que no participa en la forma de la actividad, sino que más bien queda sujeto a ella y en su fase intermedia, es decir, no toma parte ni en la decisión ni en el resultado, que sólo se le adscribe como consumidor, es decir, a extramuros de la empresa" 30 .

Taylor, ingeniero industrial y economista, es considerado el padre de la llamada Administración Científica. En 1878 efectuó sus primeras observaciones sobre el trabajo de los operarios en una empresa de acero. Realizó una serie de estudios analíticos vinculados con los tiempos de ejecución de las tareas, los movimientos, la fatiga, la remuneración del trabajo, etc. Su principal búsqueda estaba vinculada con el establecimiento 'científico' de modos de trabajar estandarizados, y llegó a establecer -junto a otros expertos- una descripción exhaustiva de la organización racional del trabajo -O.R.T-. Para Taylor, la organización y el management deben estudiarse y tratarse científicamente y no empíricamente. La improvisación tiene que ceder al planeamiento y a la ciencia. Sus estudios publicados en $1903^{31}$ originaron una revolución mental, pues en ellos se describe cómo tiene que ser el trabajador funcional. Su Escuela de Administración Científica ha sido el primer intento de hacer riguroso el saber sobre estas actividades, y puso el énfasis en la eficiencia del operario y su productividad. Estableció cuatro Principios de la Administración Científica: Planificación, Preparación, Ejecución y Control. Entre los puntos que se

29 Münch, L. (2007), pp. 75-76.

30 Polo, L. (2015), p. 45.

31 Taylor, F. (1903) y Taylor, F. (1982). 
desarrollan en la ORT $^{32}$ (organización racional del trabajo), explicita cuál es su concepción del hombre como homo oeconomicus (sólo motivado por la recompensa material), con una visión negativa del operario como irresponsable, holgazán y negligente ${ }^{33}$. Desarrolla un sistema educativo basado en la intensificación del ritmo de trabajo para lograr la eficiencia empresarial. Es innegable que ha sido el primero en llevar a cabo un análisis completo del trabajo, establecer patrones de ejecución y adiestramiento, asumiendo una actitud metódica al analizar y organizar la unidad fundamental de cualquier estructura, desde la base hasta la cima de la organización ${ }^{34}$.

Dos de sus más importantes seguidores fueron Emerson $(1853-1931)^{35} \mathrm{y}$ Henry Ford (1863-1947) ${ }^{36}$, quien como mecánico funda la Ford Motor Co., fabricando un modelo de automóvil a precios accesibles dentro de un plan de ventas y de asistencia técnica de gran alcance, revolucionando la estrategia comercial de la época. Con la aplicación que él hizo de la ORT logró establecer la línea de montaje, que permitió una producción en serie o en masa. Es el moderno método para la fabricación en las grandes fábricas de enormes cantidades de productos estandarizados.

La obra de Taylor y de sus seguidores es susceptible de numerosas y duras críticas, sin olvidar su mérito por ser pionera. De hecho Polo escribe que "una sociedad industrial distingue lo que en ella es realmente valioso, de lo que acontece en la gran cadena de la producción planificada al modo de Taylor, en la que cada uno hace lo que le toca hacer en su momento, pero no sabe nada del producto total porque se trata de actividades parciales y automáticas; y la prueba es que el hombre es reemplazable por el autómata, con lo que se cumple la observación de Aristóteles según la cual si los telares funcionaran solos, no harían falta esclavos" ${ }^{37}$. Vamos a mencionar los ocho puntos principales que se le cuestionan ${ }^{38}$. Son aspectos de su planteamiento que pesan luego

32 Taylor, F. (1982), pp. 143-150.

33 Taylor, F. (1982), p. 139.

34 Taylor, F. (1982), p. 143.

35 Emerson publicó dos libros: (1909), Efficiency as a Basis of Operation and Wages, The Engineering Magazine Co, New York, y (1912), The Twelve Principles of Efficiency, The Engineering Magazine Co., New York.

36 Ford, H. (1923).

37 Polo, L. (2015), p. 340.

38 Kantz, R. \& Kahn, D. (1970), Psicología de las Organizaciones, Edit. Atlas, Sao Paulo, pp. 92 y ss. Worthy, J, (1950), "Organizational Structure and Employee Morale", American Sociological Review, v. 15, pp. 169-179; Simon, H., (1972), El comportamiento administrativo. Estudio de los procesos decisorios en la organización administrativa, Aguilar, Madrid; Etzioni, A. (1967), Organizaciones modernas, Umusa, México. 
sobre todos los avances posteriores del management en mayor o menor medida, para contraponerse a esta postura o para intentar reivindicarla incorporando alguna nueva perspectiva. 1) Visión mecanicista de la actividad empresarial o de teoría de la máquina. 2) Superespecialización del operario. 3) Visión reductiva y microscópica del hombre. 4) Ausencia de comprobación científica. 5) Enfoque incompleto de la organización. 6) Limitación del campo de aplicación, sólo a lo productivo fabril. 7) Enfoque prescriptivo y normativo. 8) Enfoque de sistema cerrado, sólo se reduce la actividad a unas pocas variables, y el comportamiento de ellas es determinista, mecánico y con una lógica inmodificable. El concepto mecanicista y materialista de la producción es el que parece describir la realidad de la acción empresarial.

En relación a lo que este autor desarrolló, y que aún en nuestros días sigue vigente, hay varios puntos que sería oportuno incorporar en relación a lo que Polo ha escrito sobre estas posiciones. En primer lugar, Polo comenta a lo que se llega con su búsqueda de la optimización. E indica que este descubrimiento de las "normas racionales para la optimización del trabajo corre a cargo de un americano llamado Taylor" 39 . Polo explica que Taylor hace la "primera formulación racional de la actividad productiva que suelen llamar "economía de escala"

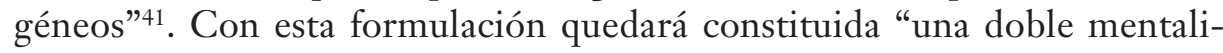
dad" 42 , la del productor y la del consumidor. La primera "dominada por la resignada aceptación de reglas racionales sin las cuales no se puede hacer nada eficazmente" ${ }^{\prime 3}$ y la segunda destinada a usar todo lo que se produzca. Se da así una materialización de la vida -hedonista y corporalista-, anclada en una ética sólo de bienes porque lo placentero, lo que el hombre tiene a su alcance son bienes inseparables de su corporeidad. Y así Polo concluye diciendo que esto conduce a que "El hombre está dividido a la manera cartesiana entre una res cogitans, que es la que produce, y una res extensa, que es la que lo pasa bien o trata de pasarlo bien. En el taylorismo se da un fuerte contraste entre la extrema especialización del productor y la globalidad del consumo" ${ }^{44}$.

Luego, en relación a cómo considera a los operarios (holgazanes, negligentes, irresponsables), Polo afirma que "la minusvaloración hunde a las per-

\footnotetext{
39 Taylor, F.W. (1911), The principles of Scientific Management, New York.

40 Polo, L. (1997), p. 125.

41 Polo, L. (1997), p. 125.

42 Polo, L. (1997), p. 125-126.

43 Polo, L. (1997), p. 126.

44 Polo, L. (1997), p. 126.
} 
sonas. La gente hace esfuerzos para merecer una mayor valoración. No hay sociedad interfamiliar sin fama, sin un sistema de ascensos en el prestigio. Un ser humano sin prestigio es un ser humano desgraciado. Desgraciado no quiere decir que lo pase mal, o que tenga una tristeza interior, sino también que su función social se debilita" ${ }^{45}$. De hecho, Polo comenta que "en una cadena de montaje taylorista, el feedback entre trabajador y empresario no se establece: la cadena de montaje no lo incluye" ${ }^{46}$.

"Con la idea de homo oeconomicus hay un déficit antropológico. El ser humano no es un individuo - un indiviso-, sino una realidad sumamente compleja, que requiere una averiguación de sus entresijos, esto es, de la conexión de sus facultades, las cuales pueden ajustarse o irse desajustando. El hombre tiene que aprender a serlo. Pero este aprendizaje puede fracasar, es decir, conducir al desajuste de las dimensiones de su ser. Dicho desajuste ocurre siempre que el hombre reduce el ámbito de sus intereses, reducción inevitable en el aislamiento que comporta la idea de individuo, el cual sólo mantiene relaciones de intercambio de medios con los demás... En cambio, la racionalización mecanicista, la mera reiteración del deseo, no son innovantes: dependen de condiciones iniciales fijas que solo permiten alcanzar un resultado homeostático. Pero el hombre no es un sistema equilibrado de esta manera, sino un ser que busca equilibrios hiperformalizados" ${ }^{47}$. Este reduccionismo en el aislamiento es una situación que se presenta frecuentemente a lo largo de todas las empresas en las que se aplica la teoría planteada por Taylor. Polo en este sentido es muy claro y contundente. Por ejemplo, escribe que "todo lo que vaya contra el crecimiento humano es simplemente malo... También lo es, por ejemplo, reducir al hombre a la condición de pieza de un sistema de montaje taylorista cuya misión es hacer coches; durante toda su vida de trabajo, a ese obrero le resultará difícil mejorar como hombre. El empresario que gerencia tal organización atenta contra el hombre: hace perder el tiempo a sus empleados y lo pierde él. La clave de la cuestión es que el hombre puede ir a más, porque nunca acaba de llegar a ser hombre; cualquier época de la vida es propicia para ser más. El hombre es un ser capaz de crecer irrestrictamente en el tiempo" 48 .

45 Polo, L. (2016), pp. 70-71.

46 Polo, L. (2015), p. 357.

47 Polo, L. (1997), p. 459.

48 Polo, L. (2016), p. 98. 
Polo sigue explicando: "Lo que intento resaltar es que la actividad económica es propia del hombre, es decir, que han de estudiarse los presupuestos antropológicos de lo económico, no vaya a ser que finjamos una figura de bomo oeconomicus separada de la integridad humana, postulada ad boc, o de acuerdo con los (supuestos) límites del método de una 'ciencia"”49.

\section{CONCLUSIÓN}

La importancia de repensar la actividad empresarial y la empresa como tal es importante, pues "El management moderno ha dado mucho, pero también se ha llevado mucho, y continúa haciéndolo. Tal vez haya llegado el momento de renegociar la oferta. Debemos aprender a coordinar los esfuerzos de miles de individuos sin crear una jerarquía opresiva de supervisores; a controlar los costes con firmeza sin asfixiar la imaginación humana, y a crear organizaciones donde la disciplina y la libertad no se excluyen mutuamente. En este nuevo siglo debemos esforzarnos por trascender las relaciones de intercambio aparentemente inevitables que forman el incómodo legado del management moderno" 50 .

En este primer escrito se ha expuesto cómo las claves y la perspectiva de Polo en relación a Frederick Taylor nos ayudan a advertir que "la actividad económica es propia del hombre, es decir, que han de estudiarse los presupuestos antropológicos de lo económico, no vaya a ser que finjamos una figura de bomo oeconomicus separada de la integridad humana, postulada ad boc, o de acuerdo con los (supuestos) límites del método de una "ciencia" ${ }^{51}$.

Se busca advertir que los autores más destacados de management, en este caso Taylor, entienden que el progreso sigue en gran parte el influjo de un concepto reductivo de racionalidad pragmática, suscrita por el cientificismo positivista $^{52}$. Nuestro empeño pasa por estudiar la conexión que existe entre teoría y práctica ${ }^{53}$, o sea, ciencia pura y ciencia aplicada. Entendiendo que en la empresa están relacionadas, no se pueden separar. Polo se calificaba de 'empresarista' pues consideraba que el cometido principal de la empresa moderna

\footnotetext{
49 Polo, L. (2015), pp. 333-334.

50 Hamel, G., (2008), p. 25.

51 Polo, L. (2015), pp. 333-334.

52 Sellés, J.F. (2011), p. 511.

53 Polo, L. (1996), p. 117.
} 
-junto a la familia y la Universidad- es hacer que la organización social sea cada vez mejor. Consideraba que la organización de la sociedad va a correr cada vez más a cargo de la empresa y si la empresa no es buena si la empresa no es humanista, si no hay virtudes, y si en los componentes de la empresa no hay virtudes; pues la empresa no cumple su cometido de institución social organizadora.

Por eso, considero de relevancia que haya nuevas generaciones de empresarios que tengan un sentido de la empresa como organización de personas, que busquen el desarrollo sostenible de la empresa como uno de los agentes de cambio de la sociedad ${ }^{54}$.

54 Cfr. Polo, L., (2015), pp. 45-46. 


\section{BIBLIOGRAFÍA}

Chiavenato, Idalberto (2012), Administración, proceso administrativo ( $3{ }^{\text {a }}$ ed.), McGraw - Hill Interamericana de México, México.

Chinchilla, Nuria (2006), Ser una empresa familiarmente responsable. Lujo o necesidad. Prentice Hall, Barcelona.

Corcuera, Juan Pablo (2001), "Naturaleza del Trabajo Directivo (una versión moderna de los temas clásicos) y su repercusión en los programas de Formación de Empresas". IESE, T-104, 01/2001. Obtenido online, febrero 2014. https://www.researchgate.net/publication/45218869_Naturaleza_del_ trabajo_directivo_una_version_moderna_de_los_temas_clasicos_y_su_repercusion_en_los_programas_de_formacion_en_direccion_de_empresas

Ferreiro, Pablo y Alcázar, Manuel (2002), Gobierno de personas en la Empresa, Ariel, Barcelona.

Ford, Henry (1923), My Life and Work, Edit. Bentham, Nueva York.

George, Claude S. Jr y Álvarez, Lourdes (2005), Historia del pensamiento administrativo, Pearson Educación, México. Obtenido online noviembre 2015: https://books.google.es/books? id=Jc8tBZb-ZJkC\&pg=PA72\&lpg= PA72\&dq=daniel + mccallum \& source $=$ bl \&ots $=1 \_9 \mathrm{~d} 4 \mathrm{udhKE} \& \operatorname{sig}=\mathrm{oKk}-$ we zbQo3QOSXjh6TNetyhHk_M\&hl=es-419\&sa=X\&ved= 0ahUKEwi2hNe79sbRAhWCQBoKHZVnBUIQ6AEIcTAQ\#v=onepage\&q=daniel $\% 20 \mathrm{mcc}$ allum \&f=false

Guillen Parra, Manuel (2000), "El lugar de la ética en la dirección de empresas. Los retos de la racionalidad", Revista Empresa y Humanismo, vol. II, ${ }^{\circ}$ 1 , pp. 75-108.

Hamel, Gary (2002), Liderando la revolución, Edit. Plume, New York.

Hamel, Gary (2009), "Moon Shots for Management", Harvard Business Review, Obtenido online julio de 2016: https://hbr.org/2009/02/moon-shotsfor-management

Hamel, Gary (2012), Lo que abora importa, Bilbao, Deusto.

Hamel, Gary y Breen, Bill (2007), El futuro del management, Harvard Business School Press, Boston.

Koontz, Harold; O’Donnell, Cyril y Weihrich, Heinz (1980), Management, Mc Graw Hill, México. 
Koontz, Harold y Weihrich, Heinz (2013), Elementos de Administración. Un enfoque internacional y de innovación, McGraw-Hill, 8va edición, México.

Koontz, Harold; Weihrich, Heinz y Cannice, Mark (1994), Administración una perspectiva global. (10 a ed.), McGraw-Hill Interamericana de México, México.

Martínez Echevarría, Miguel Alfonso (2001), Teorías de la Empresa y crisis de la modernidad, Cuadernos de Empresa y Humanismo, $\mathrm{n}^{\circ} 83$.

Martínez Echevarría, Miguel Alfonso (2002), Visiones racionalistas y románticas de la empresa, Cuadernos Empresa y Humanismo, nº 87.

Martínez Echevarría, Miguel Alfonso (2003), Los orígenes de la teoría de la empresa. Cuadernos de Empresa y Humanismo, $\mathrm{n}^{\circ} 88$.

Martínez Echevarría, Miguel Alfonso (2005), Dirigir empresas: de la teoría a la realidad, Ediciones Internacionales Universitarias, Madrid.

Martínez Echevarría, Miguel Alfonso (2015), ¿Tiene sentido una teoría de la empresa?, Cuadernos de Empresa y Humanismo, $\mathrm{n}^{\circ} 128$.

Martino, Silvia C. (2018), El aporte de Leonardo Polo a la Universidad y a la teoría de empresa, Editorial Académica Española, Mauritius.

Mintzberg, Henry (1971), "Managerial Work: Analysis from Observation”, Management Science, vol. 8, pp. 87-110.

Mintzberg, Henry (1973), The Nature of Managerial Work, New York. Harper \& Row.

Mintzberg, Henry; y Ahlstrand, Bruce y Lampel, Joseph (1999), Safari a la estrategia. Una visita guiada a través del management estratégico, Granica, Madrid.

Münch, Lourdes (2007), Administración: Escuelas, proceso administrativo, áreas funcionales y desarrollo emprendedor, Editorial Pearson, México, Madrid.

Pérez López, Juan Antonio (1970), Documento de investigación que se encuentra en el IESE, Research Paper No 5.

Pérez López, Juan Antonio (1991), Teoría de la acción bumana en las organizaciones. Colección Empresa y Humanismo, vol. 6, Madrid, Rialp.

Pérez López, Juan Antonio (1993), Fundamentos de la dirección de empresas, Rialp, Madrid. 
Polo, Leonardo (1996), Ética. Hacia una versión moderna de los temas clásicos, Aedos, Madrid.

Polo, Leonardo (2015), La esencia del hombre, Obras Completas, Serie A, vol. XXIII, Eunsa, Pamplona.

Polo, Leonardo, (2016), Quién es el Hombre, un espíritu en el tiempo, Obras Completas, Serie A, vol. X, Eunsa, Pamplona

Polo, Leonardo y Llano, Carlos (1997), Antropología de acción directiva, Unión Editorial, Madrid.

Robbins, Stephen P. (1994), Administración teoría y práctica, 4 a ed., Prentice-Hall Hispanoamericana, S.A. México.

Sellés, Juan Fernando (2011), Antropología para inconformes, Rialp, Madrid, $3^{\text {a }}$ ed.

Sellés, Juan Fernando (2013), Los tres agentes de cambio de la sociedad civil, familia, universidad y empresa, Eiunsa, Madrid.

Tzu, Sun (2009), El arte de la guerra, Ed. Mac Graw Hill Interamericana, México.

Taylor Frederick (1947), Scientific Management, Harper \& Row, New York.

Taylor Frederick [1903 (1982)], Principios de la Administración Cientifica, El Ateneo, Buenos Aires. Obtenido online, diciembre de 2015: https://www.academia.edu/30877081/Principios_de_la_administraci\%C3\%B 3n_Cient\%C3\%ADfica_F_Taylor.pdf

Vélaz, Iñaki (1996), Motivos y motivación en la empresa, Ediciones Díaz de Santos, Madrid. 